\title{
Parallel NMR Supersequences: Ten Spectra in a Single Measurement
}

\author{
Ēriks Kupče,* Jonathan R. J. Yong, Göran Widmalm, and Tim D. W. Claridge
}

Cite This: JACS Au 2021, 1, 1892-1897

Read Online

ACCESS

Wll Metrics \& More

Article Recommendations

Supporting Information

ABSTRACT: The principles employed in parallel NMR and MRI are applied to NMR supersequences yielding as many as ten $2 \mathrm{D}$ NMR spectra in one measurement. We present a number of examples where two NOAH (NMR by Ordered Acquisition using ${ }^{1} \mathrm{H}$-detection) supersequences are recorded in parallel, thus dramatically increasing the information content obtained in a single NMR experiment. The two parallel supersequences entangled by time-sharing schemes (IPAP-seHSQC, HSQCCOSY, and HSQC-TOCSY) incorporate also modified (sequential and/or interleaved) conventional pulse schemes (modules), including HMBC, TOCSY, COSY, CLIP-COSY, NOESY, and ROESY. Such parallel supersequences can be tailored for specific

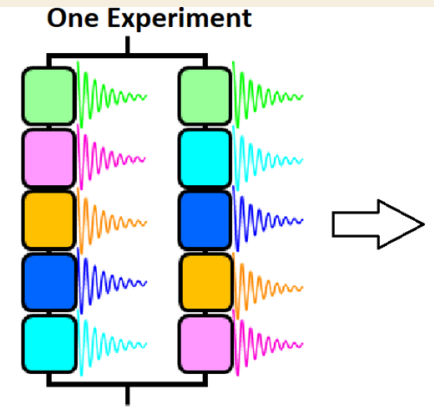

Two parallel supersequences

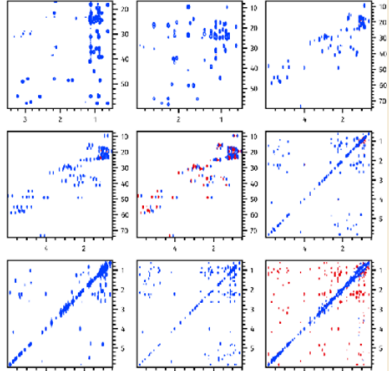

Up to 10 NMR spectra applications, for instance, the analysis and characterization of molecular structure of complex organic molecules from a single measurement. In particular, the CASPER software was used to establish the structure of a tetrasaccharide, $\beta$-LNnTOMe, with a high degree of confidence from a single measurement involving a parallel NOAH-5 supersequence.

KEYWORDS: Parallel NMR, NOAH supersequences, CASPER, CASE, oligosaccharides

N MR spectroscopy plays a fundamental role in enabling the development of new drugs and materials, and timeefficient data collection plays an increasingly important role in the analysis and characterization of these molecules by NMR. ${ }^{1-6}$ Among the more versatile approaches to this are techniques that allow recording of several spectra in a single measurement ${ }^{2,3}$ including time-shared (TS) methods, ${ }^{4}$ pulse schemes based on the PEP (Preservation of Equivalent Pathways) principles, ${ }^{5}$ the use of multiple receivers, ${ }^{6}$ and techniques based on isotope filtering concepts. ${ }^{7,8}$ The NOAH (NMR by Ordered Acquisition using ${ }^{1} \mathrm{H}$-detection) approach employs several such techniques to combine specially adapted pulse schemes (modules) into supersequences, ${ }^{9}$ allowing multiple two-dimensional (2D) spectra to be recorded using a single recovery delay $\left(d_{1}\right)$, which is by far the longest pulse sequence element in most NMR experiments. To date, up to five different experiments have been combined into a single supersequence, leading to considerable reductions in experiment time and improved sensitivity, data quality, and consistency. ${ }^{10}$ Herein, we report how the capabilities of $\mathrm{NOAH}$ supersequences may be multiplied by adapting the principles employed in parallel NMR and MRI. ${ }^{2}$ This parallelization has enabled the recording of ten $2 \mathrm{D}$ spectra within a single experiment, thereby greatly increasing the information content of NMR measurements. The parallel $\mathrm{NOAH}(p-\mathrm{NOAH})$ technique also provides for increased flexibility in tailoring supersequences for particular applications, e.g., identification of an oligosaccharide where different combinations of modules may be favored. When analyzed with a CASE (computer-assisted structure elucidation) program, CASPER, ${ }^{11}$ the structure of a tetrasaccharide was established with high confidence from five spectra obtained with a $p$ NOAH-5 supersequence in a single experiment.

One of the first experiments designed for the purpose of "black box"-style structure elucidation from a single NMR measurement was the PANACEA supersequence, which combines the HSQC, HMBC, and INADEQUATE pulse schemes into a single entity. ${ }^{12}$ The main drawback of the PANACEA experiment is the extremely low sensitivity of the direct detection of ${ }^{13} \mathrm{C}-{ }^{13} \mathrm{C}$ correlations at natural isotopic abundance (roughly one molecule in $10^{4}$ ). The NOAH supersequences exploit solely ${ }^{1} \mathrm{H}$ detection and, when combined with CASE software, ${ }^{13}$ achieve the same result in a considerably shorter period of time. ${ }^{14}$ These supersequences have been tailored for specific applications, such as metabolomics research ${ }^{10 a}$ or structure elucidation of small organic molecules. ${ }^{14}$ However, due to the limited spectral dispersion, reliable structure elucidation in more complex molecules typically requires additional information obtained by recording supplementary $2 \mathrm{D} \mathrm{NMR}$ spectra. One such example

Received: September 25, 2021

Published: November 9, 2021 
is carbohydrates, the most abundant natural products on planet Earth, which are predominantly found as polysaccharides but also as oligosaccharides and glycoconjugates. ${ }^{15}$ Linked carbohydrate monomers, referred to as glycans, may present highly complex structures including both extended linear and branched chains. These pose severe challenges for assignment, meaning a suite of NMR experiments is often required for the structure elucidation of glycans, including HSQC, HMBC, HSQC-TOCSY, HSQC-COSY, HMQC-COSY (H2BC), COSY, TOCSY, and NOESY or ROESY. ${ }^{16}$ The large number of required spectra poses a challenge for the NOAH approach that has been limited to five modules so far.

To overcome this restriction, we introduce parallel NOAH supersequences that allow combining essentially any number of modules. To illustrate this, we describe $p$-NOAH supersequences designed to acquire all the spectra required for CASE studies of oligosaccharides in a single measurement, with potential applications also to polysaccharides or glycoconjugate molecules. The recorded spectra are then analyzed using the CASPER program developed for CASE studies of complex oligo- and polysaccharides. ${ }^{11}$ CASPER predicts ${ }^{1} \mathrm{H}$ NMR and ${ }^{13} \mathrm{C}$ NMR chemical shifts based on chemical shift displacements, which depend on geometrical arrangements of the sugar residues, also referred to as increment rules. ${ }^{17}$ The input data to CASPER includes sugar components, which may have been obtained by NMR analysis, ${ }^{18}$ and unassigned NMR chemical shift data with information from up to five different 2D NMR experiments. Subsequent comparison between experimental and predicted NMR chemical shifts provides a ranking of proposed oligo- or polysaccharide structures.

The proposed approach for designing parallel NOAH supersequences is unveiled schematically in Figure 1. We start with the conventional NOAH-4 $\left(\mathrm{BS}^{\mathrm{x}} \mathrm{S}^{\mathrm{J}} \mathrm{T}\right)$ supersequence (Figure 1a) which combines the HMBC, HSQC-X $\left(S^{X}, X=\right.$ COSY or TOCSY), ${ }^{13} \mathrm{C}-{ }^{1} \mathrm{H} \quad J$-coupled and sensitivityenhanced HSQC (herein referred to as J-HSQC, $\mathrm{S}^{\mathrm{J}}$ ), and TOCSY (T) modules as previously described. ${ }^{10 \mathrm{~b}}$ The conventional HSQC-X spectra contain peaks from both the one-bond $\mathrm{C}-\mathrm{H}$ HSQC correlations, as well as $\mathrm{C}-\mathrm{H}-\mathrm{H}$ (COSY or TOCSY) correlations. In complex molecules with crowded spectra, the spectral overlap can be reduced by separating the $\mathrm{C}-\mathrm{H}$ and $\mathrm{C}-\mathrm{H}-\mathrm{H}$ correlations using spectral editing techniques, such as time-shared acquisition (Figure 1b). ${ }^{4}$ The latter approach is preferred to the original implementation ${ }^{19}$ where the HSQC spectrum is recorded as a separate reference experiment. Likewise, the TS HSQC$\operatorname{COSY}\left(\mathrm{S}^{\mathrm{C}} / \mathrm{S}\right)^{10 a}$ module, discussed in detail in the Supporting Information (SI), is preferred to otherwise similar TS HMQC$\operatorname{COSY}\left(2 \mathrm{BOB}, \mathrm{M}^{\mathrm{C}} / \mathrm{M}\right)$ module $^{8 \mathrm{a}, 14 \mathrm{~b}}$ because of the restrictions imposed by the constant-time $t_{1}$ evolution in the latter.

Just like in multiple-receiver NMR, we distinguish between three types of data acquisition modes: parallel, sequential, and interleaved. ${ }^{2,6}$ The time-shared NOAH modules acquire two spectra simultaneously (i.e., in parallel). However, unlike in multireceiver experiments, the resonances that belong to different data sets must be phase encoded so that they can be separated at the processing stage. The sequential and interleaved data are acquired in a similar fashion to the corresponding multiple-receiver experiments.

It is worth noting that the number of peaks in the J-coupled HSQC spectrum is doubled due to the ${ }^{1} J_{\mathrm{CH}}$ splitting, which
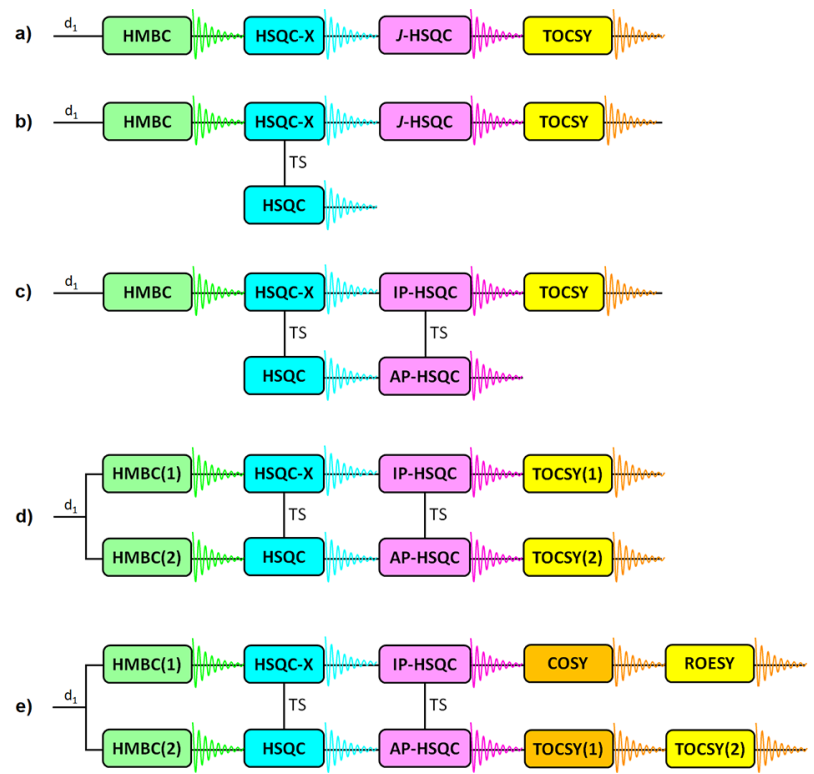

Figure 1. Schematic representation of parallel NOAH-supersequences explored in this work. Various versions of pulse schemes for individual generic modules have been described in detail elsewhere, ${ }^{5 \mathrm{~d}, 9,10,14}$ and the notation is explained in ref 24. (a) Conventional NOAH-4 $\left(\mathrm{BS}^{\mathrm{X}} \mathrm{S}^{\mathrm{I}} \mathrm{T}\right)$ supersequence $(\mathrm{X}=\mathrm{COSY}$ or TOCSY). (b) $p$-NOAH-5 $\left(\mathrm{BS}^{\mathrm{X}} \mathrm{SS}^{\mathrm{T}} \mathrm{T} / \mathrm{S}\right)$ supersequence containing the time-shared HSQC-X and HSQC module. (c) $p$-NOAH-6 $\left(\mathrm{BS}^{\mathrm{X}} \mathrm{S}^{\mathrm{IP}} \mathrm{T} / \mathrm{SS}^{\mathrm{AP}}\right)$ supersequence containing two time-shared modules $\mathrm{S}^{\mathrm{X}} / \mathrm{S}$ (HSQC-X) and $\mathrm{S}^{\mathrm{IP}} / \mathrm{S}^{\mathrm{AP}}$ (IPAP-HSQC). (d) $p$-NOAH-8 (BS $\left.{ }^{\mathrm{X}} \mathrm{S}^{\mathrm{IP}} \mathrm{T} / \mathrm{BSS}^{\mathrm{AP}} \mathrm{T}\right)$ supersequence consisting of two parallel NOAH-4 supersequences. HMBC and TOCSY spectra are recorded with two different ${ }^{n} J_{\mathrm{CH}}$-evolution and mixing times, respectively. (e) $p$-NOAH-10 (BS $\left.{ }^{\mathrm{X}} \mathrm{S}^{\mathrm{IP}} \mathrm{CR} / \mathrm{BSS}^{\mathrm{AP}} \mathrm{TT}\right)$ supersequence consisting of two parallel NOAH-5 supersequences. HMBC and TOCSY spectra are recorded with two different ${ }^{n} \mathrm{C}_{\mathrm{CH}^{-}}$ evolution and mixing times, respectively.

significantly increases the chances of spectral overlap in already crowded HSQC spectra of oligosaccharides. This problem can be addressed by replacing the J-HSQC module with a TS IPAP (inphase/antiphase) HSQC module (Figure 1c). Note that TS experiments typically require doubling the number of scans with every TS step. In this case, however, the phase encoding that allows separating the individual spectra in TS experiments can be implemented in the two TS modules independently. Thus, the minimum number of scans in this $p$-NOAH-6 $\left(\mathrm{BS}^{\mathrm{X}} \mathrm{S}^{\mathrm{IP}} \mathrm{T} / \mathrm{SS}^{\mathrm{AP}}\right.$ ) supersequence (Figure 1c) is the same as that in the $p$-NOAH-5 experiment (Figure $1 \mathrm{~b}$ ) discussed earlier. Furthermore, the conventional (non-TS) modules in such supersequences can be recorded with either double resolution or twice the number of scans. ${ }^{14 \mathrm{~b}}$ For instance, the spectra in Figure 2 were recorded with twice the number of scans in the less sensitive HMBC module and with double resolution in the high sensitivity but more crowded 2D TOCSY spectra. A similar example recorded using the $p$-NOAH-6 supersequence is shown in the SI (Figure S5). Alternatively, the non-TS modules can be recorded with two different settings, highlighting the greater versatility afforded through parallelization. For instance, the HMBC module can be recorded with two different ${ }^{n} J_{\mathrm{CH}}$-evolution delays (see Figure 1d). Acquiring several spectra with different $J$-evolution delays is common practice with conventional HMBC experiments and provides additional correlation information by sampling a wider range of ${ }^{n} J_{\mathrm{CH}}$. Likewise, it is not uncommon to record TOCSY spectra 


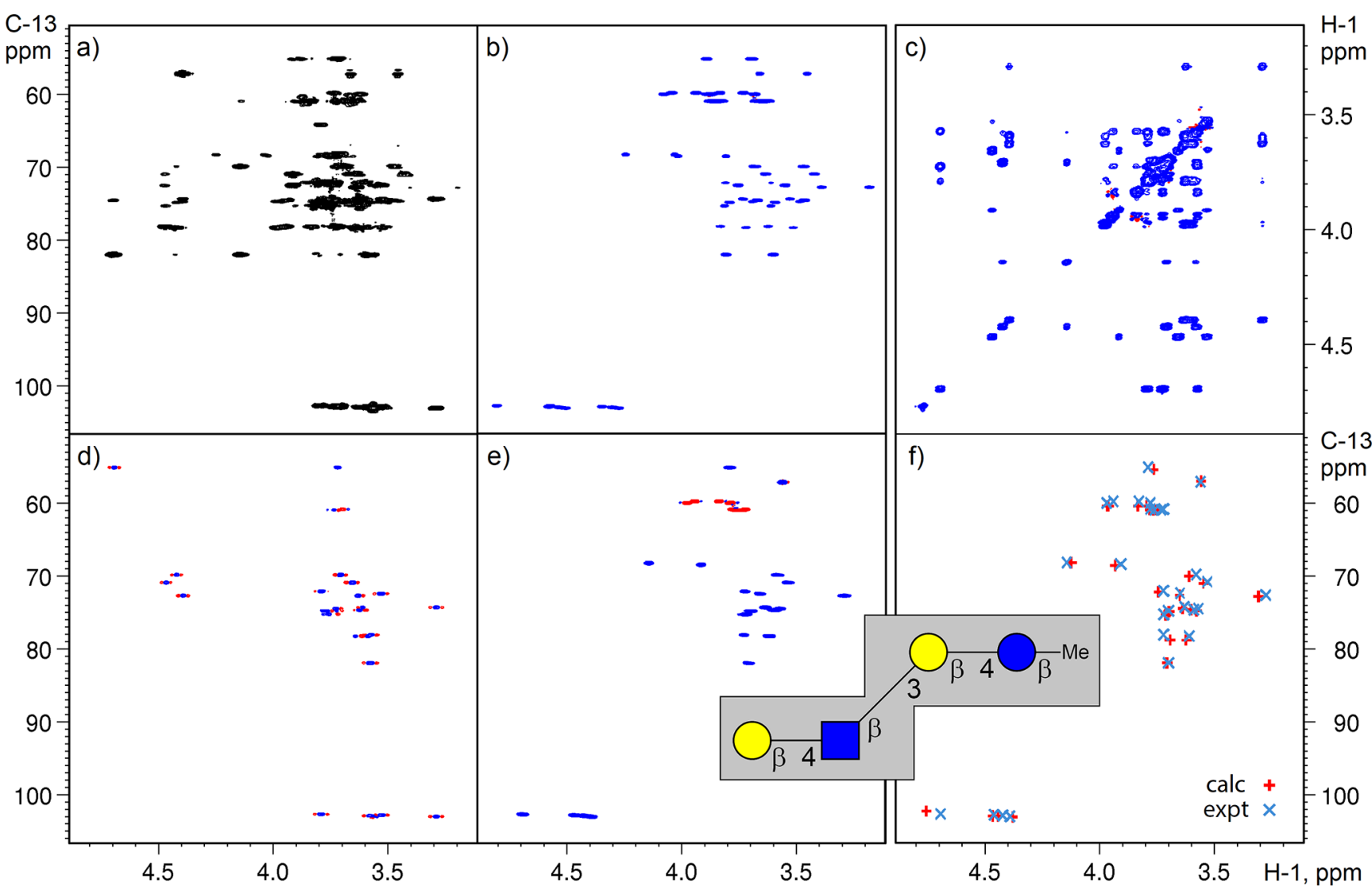

Figure 2. Spectra of $\beta \mathrm{LNnTOMe}\left(30 \mathrm{mM}\right.$ in $\left.\mathrm{D}_{2} \mathrm{O}\right)$ recorded with the $p$-NOAH-5 $\left(\mathrm{BS}^{\mathrm{C}} \mathrm{S}^{\mathrm{J}} \mathrm{T} / \mathrm{S}\right.$ ) supersequence of Figure $1 \mathrm{~b}$ : (a) HMBC, (b) $\mathrm{J}$ HSQC, (c) TOCSY, (d) multiplicity-edited HSQC-COSY, (e) multiplicity-edited HSQC, and (f) calculated vs experimental ${ }^{1} \mathrm{H}$ and ${ }^{13} \mathrm{C}$ chemical shifts from the CASPER report; the inset shows a schematic representation of the tetrasaccharide in SNFG format. ${ }^{23}$ Positive peaks are shown in blue, negative peaks in are shown red, and magnitude mode spectra are shown in black. Full spectra that include Me and CO resonances along with experimental details are provided in Figure S4.

with two or more mixing times to gain additional insight into the propagation of magnetization along the ${ }^{1} \mathrm{H}-{ }^{1} \mathrm{H}$ coupling networks. ${ }^{\text {Sd }}$ Further examples of eight spectra recorded in a single experiment are shown in the SI and listed in Table 1 together with their time savings relative to conventional experiments.

At this point, it becomes obvious that the $p$-NOAH-8 experiment in Figure $1 \mathrm{~d}$ can be seen as two NOAH-4 supersequences entangled by the phase encoding requirements in the TS modules, thus highlighting the similarity with parallel data acquisition in multiple receiver experiments. ${ }^{6}$ The $p$ NOAH-8 supersequences can be extended even further by introducing sequential NOAH modules, for instance, COSY + NOESY $(\mathrm{CN})^{5 \mathrm{~b}, \mathrm{c}}$ and TOCSY+TOCSY (TT) ${ }^{5 \mathrm{~d}}$ Once again, such modules can be implemented with either double resolution or twice the number of scans. Alternatively, the non-TS modules can be interleaved with other NOAH modules, either sequential or independent. This is shown in Figure 1e, where all types of acquisition (parallel, sequential, and interleaved) are used in the two parallel branches of the $p$ NOAH-10 ( $\left.\mathrm{BS}^{\mathrm{X}} \mathrm{S}^{\mathrm{IP}} \mathrm{CR} / \mathrm{BSS}^{\mathrm{AP}} \mathrm{TT}\right)$ supersequence. The corresponding spectra of cyclosporine $\mathrm{A}$ recorded using this supersequence are shown in Figure 3. Unlike in the $p$ NOAH-8 scheme (Figure 1d), here the two TOCSY spectra with different mixing times are implemented sequentially (Figure 1e), highlighting the distinction between the interleaved and sequential implementation of NOAH modules. This allows recording of the COSY+ROESY pair in the parallel
Table 1. Parallel NOAH Supersequences Explored in This Work $^{a}$

$\begin{array}{llllll}\text { no. } & \text { supersequence } & \text { sequence 1 } & \text { sequence 2 } & \text { spectra } & \sigma_{\mathrm{t}} \\ 1 & p \text {-NOAH-5 } & \mathrm{BS}^{\mathrm{C}} \mathrm{S}^{\mathrm{I}} \mathrm{T} & \mathrm{S} & \text { Figure 2 } & 3.6 \\ 2 & p \text {-NOAH-6 } & \mathrm{BS}^{\mathrm{C}} \mathrm{S}^{\mathrm{IP}} \mathrm{T} & \mathrm{SS}^{\mathrm{AP}} & \text { Figure S5 } & 4.0 \\ 3 & p \text {-NOAH-7 } & \mathrm{BS}^{\mathrm{C}} \mathrm{S}^{\mathrm{IP}} \mathrm{T} & \mathrm{BSS}^{\mathrm{AP}} & \text { Figure S6 } & 4.3 \\ 4 & p \text {-NOAH-8 } & \mathrm{BS}^{\mathrm{C}} \mathrm{S}^{\mathrm{IP}} \mathrm{T} & \mathrm{BSS}^{\mathrm{AP}} \mathrm{T} & \text { Figure S7 } & 4.2 \\ 5 & p \text {-NOAH-8 } & \mathrm{BS}^{\mathrm{C}} \mathrm{S}^{\mathrm{IP}} \mathrm{C}^{\mathrm{c}} & \mathrm{BSS}^{\mathrm{AP}} \mathrm{T} & \text { Figure S8 } & 4.5 \\ 6 & p \text {-NOAH-8 } & \mathrm{BS}^{\mathrm{T}} \mathrm{S}^{\mathrm{IP}} \mathrm{C}^{\mathrm{c}} & \mathrm{BSS}^{\mathrm{AP}} \mathrm{T} & \text { Figure S9 } & 4.3 \\ 7 & p \text {-NOAH-8 } & \mathrm{BS}^{\mathrm{C}} \mathrm{S}^{\mathrm{IP}} \mathrm{C}^{\mathrm{c}} & \mathrm{BSS}^{\mathrm{AP}} \mathrm{T} & \text { Figure S10 } & 4.4 \\ 8 & p \text {-NOAH-8 } & \mathrm{BS}^{\mathrm{Cc}} \mathrm{S}^{\mathrm{IP}} \mathrm{R} & \mathrm{BSS}^{\mathrm{AP}} \mathrm{T} & \text { Figure S11 } & 4.2 \\ 9 & p \text {-NOAH-9 } & \mathrm{BS}^{\mathrm{C}} \mathrm{S}^{\mathrm{IP}} \mathrm{CR} & \mathrm{SS}^{\mathrm{AP}} \mathrm{TT} & \text { Figure S12 } & 4.7 \\ 10 & p \text {-NOAH-10 } & \mathrm{BS}^{\mathrm{C}} \mathrm{S}^{\mathrm{IP}} \mathrm{CR} & \mathrm{BSS}^{\mathrm{AP}} \mathrm{TT} & \text { Figure 3 } & 4.8 \\ 11 & p \text {-NOAH-10 } & \mathrm{BS}^{\mathrm{C}} S^{\mathrm{IP}} \mathrm{CN} & \mathrm{BSS}^{\mathrm{AP}} \mathrm{TT} & \text { Figure S14 } & 4.6 \\ 12 & \text { NOAH-4 } & \mathrm{BSTT} & & \text { Figure S15 } & 2.9\end{array}$

a Abbreviations: HMBC (B), HSQC (S), COSY (C), ROESY (R), NOESY $(N)$, J-coupled HSQC $\left(S^{J}\right)$, TS IPAP-HSQC $\left(S^{I P} / S^{A P}\right)$, CLIP-COSY $\left(\mathrm{C}^{\mathrm{c}}\right)$, HSQC-COSY $\left(\mathrm{S}^{\mathrm{C}}\right)$, HSQC-TOCSY $\left(\mathrm{S}^{\mathrm{T}}\right)$, HSQCCLIP-COSY $\left(S^{C c}\right)$, time saving factor $\left(\sigma_{t}\right)$ calculated as the sum of experiment times of conventional experiments divided by the experiment time of NOAH; experimental details are given in the SI.

branch of this $p$-NOAH-10 supersequence. With a single scan per $t_{1}$ increment, the ten $2 \mathrm{D}$ spectra can be recorded in as little as $9 \mathrm{~min} 34 \mathrm{~s}$ (Figure S13). While this $p$-NOAH-10 experiment has not been tailored specifically for polypeptides, it further demonstrates the flexibility of the proposed technique and the 


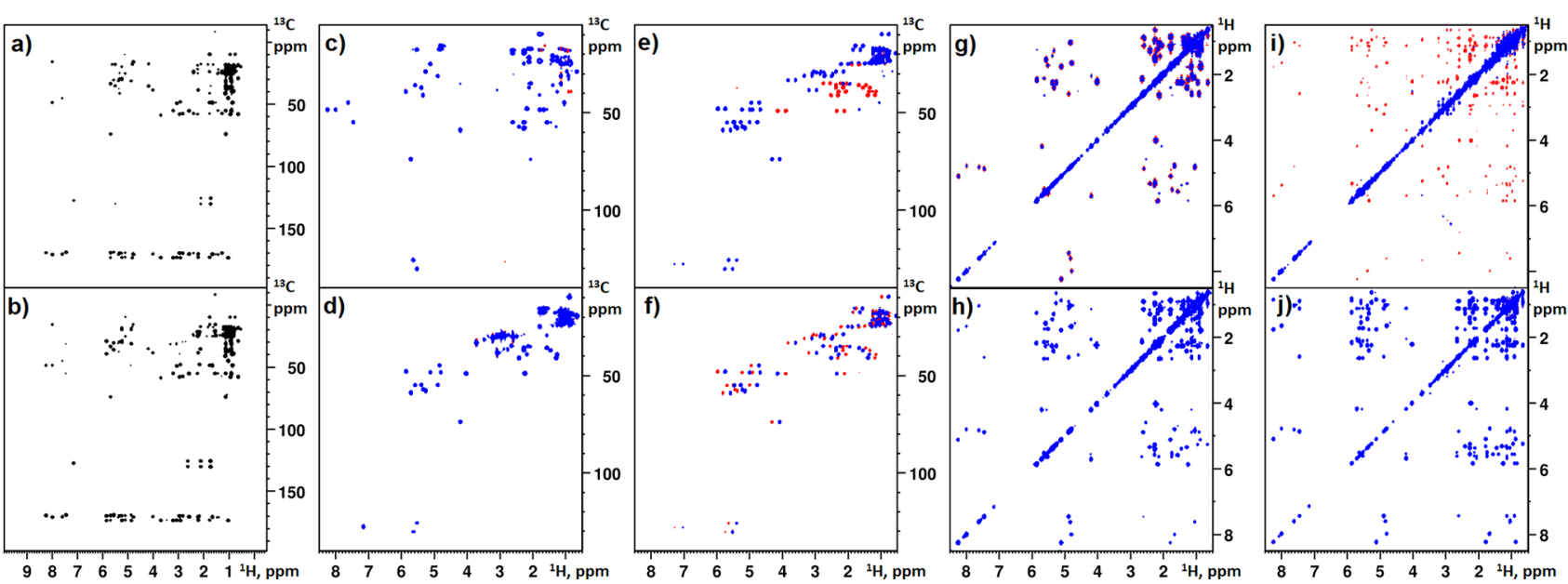

Figure 3. Spectra of cyclosporine A ( $50 \mathrm{mM}$ in benzene- $\left.d_{6}\right)$ recorded with the $p-\mathrm{NOAH}-10\left(\mathrm{BS}^{\mathrm{C}} \mathrm{S}^{\mathrm{IP}} \mathrm{CR} / \mathrm{BSS}^{\mathrm{AP}} \mathrm{TT}\right)$ supersequence shown in Figure le: (a) HMBC $\left({ }^{n} J_{\mathrm{CH}}=10 \mathrm{~Hz}\right)$, (b) HMBC $\left({ }^{n} J_{\mathrm{CH}}=5 \mathrm{~Hz}\right),(\mathrm{c})$ HSQC-CLIP-COSY, (d) HSQC, (e) multiplicity-edited IP-seHSQC, (f) multiplicity-edited AP-seHSQC, (g) COSY, (h) TOCSY (mixing time $15 \mathrm{~ms}$ ), (i) ROESY (mixing time $132 \mathrm{~ms}$ ), and (j) TOCSY (mixing time 60 $\mathrm{ms}$ ). Spectra were recorded with two scans per increment in $18 \mathrm{~min} 29 \mathrm{~s}$ on a Bruker NEO spectrometer operating at $600 \mathrm{MHz}$ and equipped with a room-temperature TXI probe. Positive peaks are shown in blue, negative peaks are shown in red, and magnitude mode spectra are shown in black. Further experimental details are provided in the SI.

many possibilities of incorporating various pulse schemes in the parallel NOAH experiments. For instance, if required, the ROESY module can easily be replaced with the NOESY module (Table 1). Likewise, the COSY and HSQC-COSY modules can be replaced with the corresponding CLIP (clean in-phase) versions (Figures S2, S3, S5-S7, S10, and S11). ${ }^{19,22}$

The five 2D NMR spectra recorded in a single $p$-NOAH-5 $\left(B S^{\mathrm{C}} \mathrm{S}^{\mathrm{J}} \mathrm{T} / \mathrm{S}\right.$ ) experiment (see Figure 2) contain optimal information for input to CASPER $^{11,20}$ using unassigned spectra. Particularly important are the TOCSY correlations with the anomeric protons and the three-bond HMBC correlations which reveal the trans-glycosidic connectivities. In the analysis of the tetrasaccharide $\beta$-D-Galp- $(1 \rightarrow 4)-\beta$-DGlc $p$ NAc- $(1 \rightarrow 3)-\beta-\mathrm{D}-\mathrm{Gal} p-(1 \rightarrow 4)-\mathrm{D}-\mathrm{Glc} p-\mathrm{OMe}$ $(\beta \mathrm{LNnTOMe})$, the methyl glycoside of a human milk oligosaccharide, ${ }^{21}$ the $N$-acetyl-D-glucosamine, methyl D-glucoside, and two D-galactose units were entered as sugar components into CASPER together with NMR data but linkage positions were given as wild cards; i.e., no information on the linkages, anomeric configurations, or sequences between sugar residues was given.

The CASPER output consists of ranked structures, with the top one given a relative score of unity and higher scores representing structures of lower compatibility. Using information from the HSQC, J-HSQC, and HSQC-COSY experiments, the relative difference to the second-best structure is 1.72. With HMBC data added, this difference increases to 2.15, and further including the TOCSY data results in a relative difference of 2.17. A relative difference of $\sim 1.2$ or larger typically suggests that the deviation between the highest ranked structure and the second best is large enough to confidently conclude the structure of an oligo- or polysaccharide based on NMR data and predictions made by the CASPER program. Detailed analysis of NMR chemical shift data from experiment and predictions can be produced as optional reports (see the SI). Thus, the $p$-NOAH-5 (BS ${ }^{\mathrm{C}} \mathrm{J}^{\mathrm{T}} \mathrm{T}$ $S$ ) supersequence tailored for use with the CASPER approach effectively provides all the required NMR data in a single measurement.
In conclusion, we have introduced a way to design parallel NMR supersequences that can be tailored for achieving specific tasks, such as analysis and structure elucidation of complex organic molecules from a single measurement. As an example, the structure of the tetrasaccharide $\beta$ LNnTOMe was established from a single measurement with a high degree of confidence using the CASPER software and the $p$-NOAH-5 $\left(B S^{\mathrm{C}} \mathrm{S}^{\mathrm{J}} \mathrm{T} / \mathrm{S}\right)$ supersequence. The combined use of interleaved, parallel (time-shared), and sequential data acquisition techniques for designing parallel experiments containing up to (but not limited to) ten modules has been demonstrated and discussed in detail. Further expansion of parallel NOAH schemes is possible by involving multiple receivers ${ }^{14 \mathrm{~b}}$ and ${ }^{15} \mathrm{~N}$ based modules. $^{9,10 \mathrm{~b}}$ The wide range of techniques available for designing parallel NMR supersequences opens new avenues for increasing the efficiency and throughput in NMR of complex organic molecules.

\section{ASSOCIATED CONTENT}

\section{Supporting Information}

The Supporting Information is available free of charge at https://pubs.acs.org/doi/10.1021/jacsau.1c00423.

Experimental details, description of HSQC-COSY modules, $p$-NOAH-5 spectra for CASPER analysis and CASPER report, spectra recorded with $p$-NOAH supersequences listed in Table 1 (PDF)

\section{AUTHOR INFORMATION}

\section{Corresponding Author}

Ëriks Kupče - Bruker UK Ltd, R\&D, Coventry CV4 9GH, United Kingdom; 이이.org/0000-0002-2366-0269;

Email: eriks.kupce@bruker.com

\section{Authors}

Jonathan R. J. Yong - Department of Chemistry, University of Oxford, Oxford OX1 3TA, United Kingdom 
Göran Widmalm - Department of Organic Chemistry, Arrhenius Laboratory, Stockholm University, S-106 91 Stockholm, Sweden; (1) orcid.org/0000-0001-8303-4481

Tim D. W. Claridge - Department of Chemistry, University of Oxford, Oxford OX1 3TA, United Kingdom; (1) orcid.org/ 0000-0001-5583-6460

Complete contact information is available at: https://pubs.acs.org/10.1021/jacsau.1c00423

\section{Notes}

The authors declare no competing financial interest.

\section{ACKNOWLEDGMENTS}

Dedicated to Professor Ray Freeman on the occasion of his 90th birthday. This work was supported by grants to G.W. from the Swedish Research Council (2017-03703) and The Knut and Alice Wallenberg Foundation. J.R.J.Y. thanks the Clarendon Fund (University of Oxford) and the EPSRC Centre for Doctoral Training in Synthesis for Biology and Medicine (EP/L015838/1) for a studentship, generously supported by AstraZeneca, Diamond Light Source, Defense Science and Technology Laboratory, Evotec, GlaxoSmithKline, Janssen, Novartis, Pfizer, Syngenta, Takeda, UCB, and Vertex.

\section{REFERENCES}

(1) Rouger, L.; Gouilleux, B.; Giraudeau, P. Fast n-Dimensional Data Acquisition Methods. In Encyclopedia of Spectroscopy and Spectrometry; Lindon, J. C., Tranter, G. E., Koppenaal, D. W., Eds; Academic Press, 2017; pp 588-596.

(2) (a) Kupče, $\bar{E}$.; Mote, K. R.; Webb, A.; Madhu, P. K.; Claridge, T. D.W. Multiplexing Experiments in NMR and Multi-Nuclear MRI. Prog. Nucl. Magn. Reson. Spectrosc. 2021, 124-125, 1-56. (b) Kupče, E.; Frydman, L.; Webb, A. G.; Yong, J. R. J.; Claridge, T. D. W. Parallel Nuclear Magnetic Resonance (NMR) Spectroscopy. Nat. Rev. Methods Primers 2021, 1, 27.

(3) (a) Das, B. B.; Opella, S. J. Multiple acquisition/multiple observation separated local field/chemical shift correlation solid-state magic angle spinning NMR spectroscopy. J. Magn. Reson. 2014, 245, 98-104. (b) Gopinath, T.; Veglia, G. Chapter Four - Simultaneous acquisition of multiple fast MAS solid-state NMR experiments via orphan spin polarization. Annu. Rep. NMR Spectrosc. 2021, 102, 247268. (c) Gallo, A.; Franks, W. T.; Lewandowski, J. R. A suite of solidstate NMR experiments to utilize orphaned magnetization for assignment of proteins using parallel high and low gamma detection. J. Magn. Reson. 2019, 305, 219-231.

(4) Parella, T.; Nolis, P. Time-shared NMR experiments. Concepts Magn. Reson., Part A 2010, 36A, 1-23.

(5) (a) Cavanagh, J.; Rance, M. Sensitivity improvement in isotropic mixing (TOCSY) experiments. J. Magn. Reson. 1990, 88, 72-85. (b) Gurevich, A. Z.; Barsukov, I. L.; Arseniev, A. S.; Bystrov, V. F. Combined COSY-NOESY experiment. J. Magn. Reson. 1984, 56, 471-478. (c) Haasnoot, C. A. G.; van de Ven, F. J. M.; Hilbers, C. W. COCONOSY. Combination of $2 \mathrm{D}$ correlated and $2 \mathrm{D}$ nuclear Overhauser enhancement spectroscopy in a single experiment. $J$. Magn. Reson. 1984, 56, 343-349. (d) Nolis, P.; Motiram-Corral, K.; Pérez-Trujillo, M.; Parella, T. Interleaved Dual NMR Acquisition of Equivalent Transfer Pathways in TOCSY and HSQC Experiments. ChemPhysChem 2019, 20, 356-360.

(6) (a) Kovacs, H.; Kupče, E. . Parallel NMR Spectroscopy With Simultaneous Detection of ${ }^{1} \mathrm{H}$ and ${ }^{19} \mathrm{~F}$ Nuclei. Magn. Reson. Chem. 2016, 54, 544-560. (b) Kupče, $\overline{\mathrm{E}}$. NMR with multiple Receivers. In Modern NMR Methodology; Heise, H., Matthews, S., Eds.; Topics in Current Chemistry; Springer: Berlin, 2011; pp 71-96.

(7) Breeze, A. L. Isotope-filtered NMR methods for the study of biomolecular structure and interactions. Prog. Nucl. Magn. Reson. Spectrosc. 2000, 36, 323-372.
(8) (a) Nagy, T. M.; Gyöngyösi, T.; Kövér, K. E.; Sørensen, O. W. BANGO SEA XLOC/HMBC-H2OBC: complete heteronuclear correlation within minutes from one NMR pulse sequence. Chem. Commun. 2019, 55, 12208-12211. (b) Nagy, T. M.; Kövér, K. E.; Sørensen, O. W. Double and adiabatic BANGO for concatenating two NMR experiments relying on the same pool of magnetization. J. Magn. Reson. 2020, 316, 106767.

(9) Kupče, E.; Claridge, T. D. W. NMR Supersequences for Small Molecule Analysis and Structure Elucidation. Angew. Chem., Int. Ed. 2017, 56, 11779-11783.

(10) (a) Hansen, A. L.; Kupče, E. E.; Li, D.-W.; Bruschweiler-Li, L.; Wang, C.; Brüschweiler, R. 2D NMR-Based Metabolomics with HSQC/TOCSY NOAH Supersequences. Anal. Chem. 2021, 93, 6112-6119. (b) Yong, J. R. J.; Hansen, A. L.; Kupče, E.; Claridge, T. D. W. Increasing Sensitivity and Versatility in NMR Supersequences with New HSQC-based Modules. J. Magn. Reson. 2021, 329, 107027.

(11) Lundborg, M.; Widmalm, G. Structural Analysis of Glycans by NMR Chemical Shift Prediction. Anal. Chem. 2011, 83, 1514-1517.

(12) (a) Kupče, E. .; Freeman, R. Molecular Structure from a Single NMR Experiment. J. Am. Chem. Soc. 2008, 130, 10788-10792. (b) Kupče, E..$;$ Freeman, R. Molecular structure from a single NMR sequence (fast-PANACEA). J. Magn. Reson. 2010, 206, 147-153.

(13) (a) Béni, Z.; Szakács, Z.; Sánta, Z. Computer-Assisted Structure Elucidation in NMR. In Anthropic Awareness; Szántay, C., Ed.; Elsevier, 2015; pp 317-354. (b) Plainchont, B.; de Paulo Emerenciano, V.; Nuzillard, J.-M. Recent advances in the structure elucidation of small organic molecules by the LSD software. Magn. Reson. Chem. 2013, 51, 447-453. (c) Elyashberg, M.; Williams, A. J.; Blinov, K. Structural revisions of natural products by ComputerAssisted Structure Elucidation (CASE) systems. Nat. Prod. Rep. 2010, 27, 1296-1328.

(14) (a) Kupče, E. .; Claridge, T. D. W. Molecular structure from a single NMR supersequence. Chem. Commun. 2018, 54, 7139-7142. (b) Kupče, E. E.; Claridge, T. D. W. New NOAH modules for structure elucidation at natural isotopic abundance. J. Magn. Reson. 2019, 307, 106568. (c) Claridge, T. D. W.; Mayzel, M.; Kupče, E. Triplet NOAH supersequences optimized for small molecule structure characterisation. Magn. Reson. Chem. 2019, 57, 946-952.

(15) Ernst, B.; Magnani, J. From carbohydrate leads to glycomimetic drugs. Nat. Rev. Drug Discovery 2009, 8, 661-677.

(16) Widmalm, G. General NMR Spectroscopy of Carbohydrates and Conformational Analysis in Solution. In Comprehensive Glycoscience, 2nd ed.; Barchi, J., Jr., Ed.; Elsevier: Oxford, 2021; Vol. 1, pp 340-373.

(17) Ståhle, J.; Widmalm, G. NMR Chemical Shift Predictions and Structural Elucidation of Oligo- and Polysaccharides by the Computer Program CASPER. In NMR in Glycoscience and Glycotechnology; Kato, K., Peters, T., Eds.; The Royal Society of Chemistry: London, 2017; pp 335-352.

(18) Lundborg, M.; Fontana, C.; Widmalm, G. Automatic Structure Determination of Regular Polysaccharides Based Solely on NMR Spectroscopy. Biomacromolecules 2011, 12, 3851-3855.

(19) Gyöngyösi, T.; Timári, I.; Haller, J.; Koos, M. R. M.; Luy, B.; Kövér, K. E. Boosting the NMR Assignment of Carbohydrates with Clean In-Phase Correlation Experiments. ChemPlusChem 2018, 83, 53-60.

(20) Lundborg, M.; Widmalm, G. NMR Chemical Shift Prediction of Glycans: Application of the Computer Program CASPER in Structural Analysis. Methods Mol. Biol. 2015, 1273, 29-40.

(21) Maliniak, A.; Widmalm, G. Structural Analysis of Carbohydrates by Nuclear Magnetic Resonance Spectroscopy and Molecular Simulations: Application to Human Milk Oligosaccharides. In Food Oligosaccharides: Production, Analysis and Bioactivity; Javier Moreno, F., Luz Sanz, M., Eds.; John Wiley \& Sons, Ltd: Chichester, 2014; pp 320-349.

(22) (a) Gyöngyösi, T.; Timári, I.; Sinnaeve, D.; Luy, B.; Kövér, K. E. Expedited Nuclear Magnetic Resonance Assignment of Small- to Medium-Sized Molecules with Improved HSQC-CLIP-COSY Experiments. Anal. Chem. 2021, 93, 3096-3102. (b) Koos, M. R. M.; 
Kummerlöwe, G.; Kaltschnee, L.; Thiele, C. M.; Luy, B. CLIP-COSY: A Clean In-Phase Experiment for the Rapid Acquisition of COSYtype Correlations. Angew. Chem., Int. Ed. 2016, 55, 7655-7659.

(23) Neelamegham, S.; Aoki-Kinoshita, K.; Bolton, E.; Frank, M.; Lisacek, F.; Lütteke, T.; O’Boyle, N.; Packer, N. H.; Stanley, P.; Toukach, P.; Varki, A.; Woods, R. J.; The SNFG Discussion Group. Updates to the Symbol Nomenclature for Glycans guidelines. Glycobiology 2019, 29, 620-624.

(24) The single letter notation introduced for basic experiments (8) is extended to cover more complex pulse schemes (10). A capital subscript (where required) denotes nucleus, e.g., $\mathrm{S}_{\mathrm{N}}\left({ }^{15} \mathrm{~N}\right.$ HSQC) or $\mathrm{S}_{\mathrm{C}}\left({ }^{13} \mathrm{C}\right.$ HSQC). A capital superscript denotes the subtype of experiment, while the (optional) small letter denotes a particular variant. For instance, CLIP-COSY $\left(\mathrm{C}^{\mathrm{c}}\right)$ or HSQC-CLIP-COSY $\left(\mathrm{S}^{\mathrm{Cc}}\right)$. Likewise, the $\mathrm{H} 2 \mathrm{OBC}$ module is a constant-time variant of the HMQC-COSY pulse scheme and is denoted here as $\mathrm{M}^{\mathrm{C}}$ whereas the $T S$ version of the same experiment named $2 \mathrm{BOB}$ is accordingly denoted as $\mathrm{M}^{\mathrm{C}} / \mathrm{M}$. 